\title{
Large Mammals Diversity Assessment in Chilalo-Galema National Park, Ethiopia
}

\author{
Girma Lema ${ }^{1}$, Mesele Admassu ${ }^{2,}$ * \\ ${ }^{1}$ Biology Department, Dera Senior Secondary School, Dera, Ethiopia \\ ${ }^{2}$ Biology Department, Adama Science and Technology University, Adama, Ethiopia \\ Email address: \\ girma2009bio@gmail.com (G. Lema), meseleadmassu@gmail.com (M. Admassu) \\ ${ }^{*}$ Corresponding author
}

\section{To cite this article:}

Girma Lema, Mesele Admassu. Large Mammals Diversity Assessment in Chilalo-Galema National Park, Ethiopia. European Journal of Biophysics. Vol. 8, No. 2, 2020, pp. 26-34. doi: 10.11648/j.ejb.20200802.13

Received: November 27, 2019; Accepted: December 18, 2019; Published: September 24, 2020

\begin{abstract}
A study on the assessment of species diversity of large mammals was conducted in Chilalo Galema Mountain in east Arsi administrative zone from February 2019 to September 2019. The aim of this study was to assess large mammalian species diversity and human-wildlife conflict in Chilalo-Galema Mountain National Park. Line transects and observation methods were used to collect data. Twenty six transect lines were laid, varied in length from $1.41 \mathrm{~km}$ to $4.34 \mathrm{~km}$. Transect width ranged from $200 \mathrm{~m}$ to $400 \mathrm{~m}$. In this study both primary and secondary data were used. Direct observation, body parts, vocalization and indirect evidences such as footprints, burrowing and droppings were used to collect data on diversity of large mammals. Observation and direct count of animals were made during 6:00-10:00 a.m. in the morning and 3:00-6:00 p.m. in the late afternoon when most mammals were active. Species diversity of large mammals was calculated using the Shannon-Weaver index of diversity. The evenness of mammalian species was calculated as $\mathrm{J}=\mathrm{H}^{\prime} / \mathrm{H}^{\prime} \max$ where H'max $=\ln (\mathrm{s}) .28$ species that belong to seven Orders (Primates, Artiodactyla, Carnivora, Hyracoidea, Tubulidentata, Rodentia and Lagomorpha) were identified and recorded during the study. Out of the 252 recorded mammals $26(10.08 \%)$ were order primates, 50 (19.38\%) were order carnivora, $1(0.39 \%)$ were order hyracoidea, $6(2.33 \%)$ were order tubulidentata, $164(63.57 \%)$ were artiodactyla, 8 $(3.10 \%)$ were rodentia and $2(0.78 \%)$ were lagomorpha. Mammalian fauna of the Chilalo Galema Mountain was dominated by two groups of orders, the most abundant orders; namely, order artiodactyla and order carnivora, which contain 164 (63.57\%) and $50(19.38 \%)$ mammals respectively. The least abundant was order hyracoidean with $1(0.39 \%)$ recorded mammal. So, Chilalo-Galema is rich in mammalian species diversity and needs conservation measures.
\end{abstract}

Keywords: Abundance, Assessment, Chilalo-Galema, Large Mammals, Species Diversity

\section{Introduction}

About 1,150 species of mammals were described and listed from the continent of Africa [1]. East Africa is rich in mammalian fauna. Ethiopia is widely acknowledged as having high rates of endemism across all taxa including mammals. Ethiopia is rich in animal diversity for instance it harbours about 861 species of birds, 284 species of mammals, 201 species of reptiles, 63 species of amphibians and 150 species of fish [2]. Among these, 31 species of mammals, 16 species of birds, 24 species of amphibians, 9 species of reptiles and 40 species of fishes are endemic. Out of 31 species of endemic mammals, five species are larger mammals and the rest are smaller ones [3]. However, these vary from place to place and season to season due to climatic and environmental factors [4]

Ethiopia is often known as 'the roof of Africa' due to its raged topography mountainous environment. It is endowed with extensive and unique environmental conditions, ranging from the lowest altitude in Dallol depression which is 100 masl to the highest altitude at Ras Dashen which is 4620 masl [5]. The wide variety of habitats in Ethiopia, ranging from arid desert, open grassland and semi-arid savannas to highland diversity of cultures and ecology is further reflecting by forests and Afro-alpine moorlands, supports an exceptionally diverse fauna and flora. Ethiopia's uneven topography and the varied climatic conditions have endowed it with impressive wildlife species. Ethiopia is the home to various endemic, endangered, 
vulnerable and rare species [6]. Ethiopia is one of the biodiversity hotspot areas of Africa. Ethiopia is among the world's best biodiversity area in terms of richness and endemism of mammalian species. Ethiopia and Tanzania are among the top 25 endemic rich countries of the world in terms of higher vertebrate species whereas Ethiopia, Kenya, Uganda and Tanzania are individually among the world leaders in terms of richness and endemic of mammals. Those areas face many challenges due to growing population, borders conflict and drought. Mammals are often the first taxa to be listed for a site. Ethiopia contains largest concentration of large mammals within the national parks. However, complete inventory does not exist and endemism not well illustrated the species diversity for better management of biodiversity [6].

The wildlife population has been declining throughout the world at an alarming rate. This is mainly due to habitat destruction and expansion of agriculture [7-10]. This destruction is mainly pronounced in developing countries like Ethiopia. Ethiopia's increasing human population has substantially fragmented the wildlife habitat as settlement and agriculture extends into the mountain leading to habitat destruction due to overgrazing by livestock. The Ethiopian high lands are among the most densely populated agricultural areas in Africa [11]. With expansion of human habitation, many wildlife populations have become restricted and isolated in National Parks. Small fragments of habitats exist outside protected areas; however, their significance in maintaining diverse groups of species in Ethiopia is poorly understood. Therefore, accurate population estimate of these diverse wildlife forms is an essential requirement for conservation efforts. An assessment of the conservation status of all known mammals was conducted by the International Union for Conservation of Nature and Natural Resources [12]. The
IUCN Red List classifications of extinction risk have been used in numerous studies, including the identification of traits associated with high extinction risk [13-14] prediction of future species losses and prioritization of species for conservation action. However, an assessment of large mammalian species has never been carried out in ChilaoGalema National Park until now to determine the conservation status. Therefore, the current study aimed at assessing the large mammalian species at Chilalo-Galema National Park.

\section{Materials and Methods}

\subsection{Study Area}

Arsi Mountains National Park was established recently in 2011. This Park is found in eastern and western Arsi zones. The Park is subdivided in to 4 Blocks. These are DeraDilfekar, Chilalo-Galema, Kaka and Honkolo blocks. Due to the presence of these blocks, the park has different agroecological zones. It has a total area of $931 \mathrm{~km}^{2}$. Arsi Mountains National Park is about $114 \mathrm{~km}$ far away from the capital city Addis Ababa. Chilalo Galema Mountain block is found in the central part of the East Arsi Administrative Zone and lies between $7^{\circ} 28^{\prime}$ to $8^{\circ} 58^{\prime} \mathrm{N}$ latitude and $39^{\circ} 17^{\prime}$ to $39^{\circ} 28^{\prime} \mathrm{E}$ longitude (Figure 1). The block is located in the South Eastern part of Ethiopia. The total area of the block is about $792 \mathrm{~km}^{2}$ this is the largest block from the rest of the 3 blocks. Before the establishment of the park it was used as priority forest conservation and controlled hunting area. Mount Chilalo-Galema is part of the Arsi Mountains comprising the second highest afro-alpine habitat in Ethiopia [15-16].

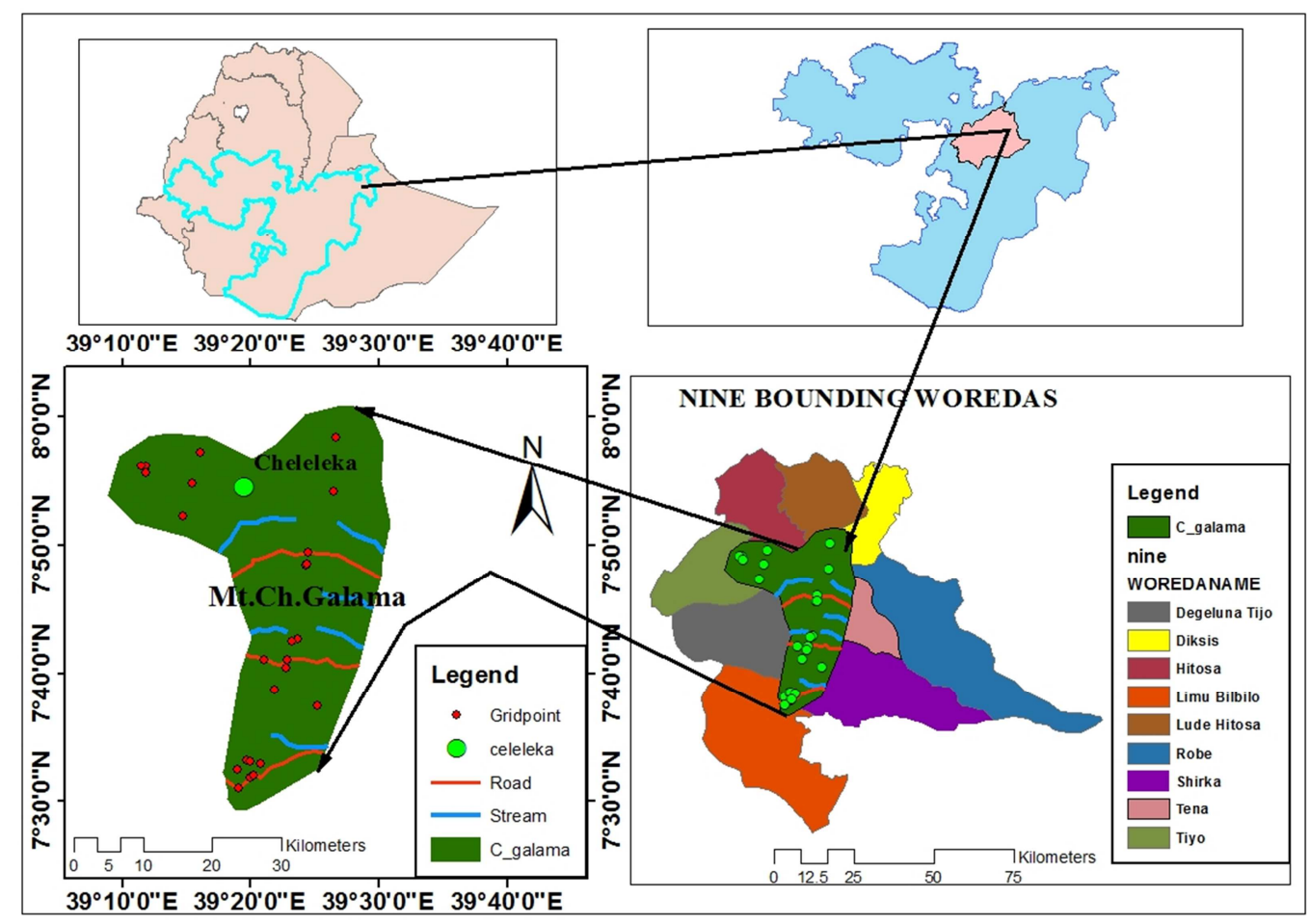

Figure 1. Map of the study area. 
Mount Chilalo-Galema is about 3,300 masl. The average annual temperature is lessan $10^{\circ} \mathrm{C}$ while areas lessan 3,300 masl show ranges from $10^{\circ} \mathrm{C}$ to $15^{\circ} \mathrm{C}$. The maximum and minimum temperature is $25.9^{\circ} \mathrm{C}$ and $8.5^{\circ} \mathrm{C}$ respectively. The hottest and coldest months of the year are March and December respectively [17]. The annual rain fall ranges from $800 \mathrm{~mm}$ to $2000 \mathrm{~mm}$ [18]. The mountain is characterized by three vegetation zones and these are Erica forest, afro-montane and afro-montane forest which has been contributing for the survival of large mammals in the area.

\subsection{Methods}

Line transect method was used to collect data on the species of large mammals from the study area. Twenty six transect lines were laid, which varied in length from $1.41 \mathrm{~km}$ to $4.34 \mathrm{~km}$. Transect width ranged from $200 \mathrm{~m}$ to $400 \mathrm{~m}$. Among 26 transect lines, ten transect lines were laid on Ericaceous forest habitat, six transect lines were laid on afroalpine forest habitat and ten transect lines were laid on afromontane (woodland) forest habitat. Each transect line was set at a distance of $500 \mathrm{~m}(0.5 \mathrm{~km})$ from other transects to avoid double counting. Two rounds of observations of large mammals during the late dry season and early wet season were carried out from February 2018 to July 2019. A total of $67 \mathrm{~km}$ distance was covered during the study. Observation was carried out along the line transect against the direction of wind to minimize disturbances [19]. Direct observation method was used to identify and record large mammals in the study area.

\subsection{Data Collection}

The present assessment was conducted during dry and wet season which are suitable to get data on large mammals [20]. To assess large mammal species diversity, direct evidences include directly observing the animal, body parts and vocalization. Indirect evidences include observing footprints, burrowing and droppings [21]. During line transect survey, actual sightings of individual or group of animals or their signs such as droppings, footprints, vocalizations and dropped body structures such as spines for porcupine were recorded. Data collected from transect lines of similar habitats were pooled together for a particular habitat for analysis. Observation and direct counts of large mammals were made during 6:00 a.m to 10:00 a.m. in the morning and 4:00 p.m to $6: 00$ p.m. in the afternoon when most mammals were active in the study area. When mammals observed, instantly recorded with their local name and the scientific name was determined later with the help of the field guide book [22].

\subsection{Data Analysis}

Encounter rate was computed as the total number of encounters for each species per total distance travelled. The relative abundance (Ar) was estimated as the total number of encounters of a species (calls, spoors and droppings) per distance of transects walked (encounter rate) at the study area [23] and [24].

Thus,

\section{$\mathrm{Ar}=\mathrm{Tse} / \mathrm{Tlt}$}

where,

Tse=total number of species encountered and

$\mathrm{Tlt}=$ total length of transect walked

The Species diversity of large mammals in ChilaloGalema Mountains was calculated using the ShannonWeaver index.

$$
\text { Shannon Index }(H)=-\sum_{i=1}^{s} \text { PilnPi }=-N P i l n P i
$$

Where $\mathrm{H}=$ Shannon diversity index

$\mathrm{Pi}=\mathrm{is}$ the proportion $(\mathrm{n} / \mathrm{N})$ of the $\mathrm{i}^{\text {th }}$ species in the habitat

$\mathrm{S}=$ Number of species encountered

$\Sigma=$ sum for species 1 to species $\mathrm{S}$

$\mathrm{ln}=$ is the natural logarism

$\mathrm{N}=$ Total number of individuals found and $(\mathrm{n})=$ Number of one particular specie found

$\mathrm{H}$ is influenced both by number of species as well as by the evenness with which mammals are distributed with those species. Equal $\mathrm{H}$ values may thus be obtained if one habitat contains fewer and evenly distributed species of mammals [25].

The evenness of mammalian species was calculated as

$$
\mathrm{J}=\mathrm{H} / \mathrm{H} \max
$$

Where $\mathrm{J}=$ evenness of mammalian species

$\mathrm{H}=$ Shannon diversity index

$\mathrm{H} \max =\ln (\mathrm{s})$

$\mathrm{s}=$ number of specie

$\mathrm{n}=$ number of individual

$\ln =$ is the natural $\log$

This measure varies between 1 (complete evenness) and 0 (complete unevenness). Chi-square $(\chi 2)$ was used to compare differences in abundance of mammal species between habitats and the overall significant difference in abundance of large-sized mammal species in the study area. SPSS computer program was used for Chi-square analysis to test the association of large mammal species and their habitats [26]. Simpson similarity index (SI) was also computed to assess the similarity among and between three habitats with reference to the composition of species.

$$
\mathrm{SI}=3 \mathrm{C} / \mathrm{I}+\mathrm{II}+\mathrm{III}
$$

Where: SI=Simpson's similarity index

$\mathrm{C}=$ number of common species to all three habitat

$\mathrm{I}=$ the number of species in habitat one

$\mathrm{II}=$ the number of species in habitat two

$\mathrm{III}=$ the number of species in habitat three 


\section{Results}

A total of 28 species that belongs to seven Orders were identified and recorded in Chilalo-Galema National Park, namely order Primates, Artiodactyla, Carnivora, Hyracoidea, Tubulidentata, Rodentia and Lagomorpha. The families under the above orders are listed in (Table 1).

Table 1. Estimated population, relative abundance and encounter rate of large mammals in Chilalo-Galema National Park.

\begin{tabular}{|c|c|c|c|c|c|c|}
\hline Order & Family & Common name & Scientfic name & $\begin{array}{l}\text { Total } \\
\text { observed }\end{array}$ & $\begin{array}{l}\text { Relative } \\
\text { abundance }\end{array}$ & $\begin{array}{l}\text { Encounter } \\
\text { rate }\end{array}$ \\
\hline \multirow{3}{*}{ Primates } & \multirow{2}{*}{ Cercopithecidae } & Anubis baboon & Papio Anubis & 13 & 0.19 & 0.19 \\
\hline & & Grivet monkey & Cercopithecus aethiops & 7 & 0.1 & 0.1 \\
\hline & Colobidae & Black-and-white colobus & Colobus guereza & 6 & 0.09 & 0.09 \\
\hline \multirow{12}{*}{ Carnivora } & \multirow{3}{*}{ Canidae } & Ethiopian wolf & Canis simensis & 14 & 0.21 & 0.21 \\
\hline & & Black-back jackal & Canis mesomelas & 4 & 0.06 & 0.06 \\
\hline & & Common jackal & Canis aureus & $*$ & $*$ & $*$ \\
\hline & Mustelidae & Zorrilla & Ictonyx striatus & $*$ & $*$ & $*$ \\
\hline & Viverridae & African civet & Civettictis civetta & 2 & 0.03 & 0.03 \\
\hline & \multirow{3}{*}{ Herpestidae } & Slender-mongoose & Herpestes sanguine & 4 & 0.06 & 0.06 \\
\hline & & Honey badger & Mellivoracapensis & $*$ & $*$ & $*$ \\
\hline & & Mongoose/Egyptia & Herpestes inchneumon & $*$ & $*$ & $*$ \\
\hline & \multirow{2}{*}{ Hyaenidae } & Spotted hyaena & Crocuta crocuta & 17 & 0.25 & 0.25 \\
\hline & & Aard wolf & Protelus cristatus & $*$ & $*$ & $*$ \\
\hline & \multirow[t]{2}{*}{ Felidae } & Leopard & Panthera pardus & 2 & 0.03 & 0.03 \\
\hline & & Serval & Felis serval & $*$ & $*$ & $*$ \\
\hline \multirow{3}{*}{$\begin{array}{l}\text { Hyracoidea } \\
\text { Tubulidentata }\end{array}$} & Procaviidae & Ethiopian rock hyrax & Procavia habessinica & $*$ & $*$ & $*$ \\
\hline & Orycteropodidae & Aardvark & Orycteropus afer & 6 & 0.09 & 0.09 \\
\hline & Suidae & Warthog & Phacochoerus africanus & 48 & 0.72 & 0.72 \\
\hline \multirow{6}{*}{ Artiodactyla } & \multirow{6}{*}{ Bovidae } & Bush duiker & Sylvicapra grimmia & 3 & 0.05 & 0.05 \\
\hline & & Klipspringer & Oreotragus oreotragus & 12 & 0.18 & 0.18 \\
\hline & & Bohor reedbuck & Redunca redunca & 70 & 1.04 & 1.04 \\
\hline & & Bush buck & Tragelaphus scriptus & 2 & 0.03 & 0.03 \\
\hline & & Menelik's bushbuck & Tragelaphus scriptus meneliki & 6 & 0.09 & 0.09 \\
\hline & & Monain nyala & Tragelaphus buxtoni & 23 & 0.34 & 0.34 \\
\hline Rodentia & Hystricidae & Porcupine & Hystrix cristata & 8 & 0.12 & 0.12 \\
\hline Lagomorpha & Leporidae & Abyssinian Hare & Lepus capensis & 2 & 0.03 & 0.03 \\
\hline
\end{tabular}

*Indicate secondary data from Chilao-Galema National Park

\subsection{Large Mammalian Species Identified and Recorded in Chilalo-Galema National Park}

Twenty- eight mammalian species were encountered during this assessment. The species identified, habitat types and methods of detection were given in (Table 2). Out of 28 mammalian species the presence of $19(67.86 \%)$ species were confirmed through direct and indirect evidence while 9 $(32.14 \%)$ species were recorded from secondary data sources of Chilalo-Galema National Park (Table 2).

Table 2. Habitat types and large mammalian species identified and recorded in Chilalo-Galema National Park.

\begin{tabular}{|c|c|c|c|c|c|c|c|}
\hline \multirow{2}{*}{ S.No } & \multirow{2}{*}{ Common name } & \multirow{2}{*}{ Local name } & \multirow{2}{*}{ Scientific name } & \multirow{2}{*}{ Methods of identification } & \multicolumn{3}{|c|}{ Habitat types } \\
\hline & & & & & Erc & Af-al & Af-mo \\
\hline 1 & Mountain nyala & Gadamsa baddaa & Tragelaphus buxtoni & Observation & 1 & 1 & 1 \\
\hline 2 & Bohor reedbuck & Godaa & Redunca redunca & Observation & 1 & 0 & 1 \\
\hline 3 & Red duiker & Kuruphee & Cephalophus natelensis & Observation & 1 & 0 & 1 \\
\hline 4 & Ethiopian wolf & Jeedala Dimtuu & Canis simensis & Observation & 1 & 1 & 0 \\
\hline 5 & Porcupine & Xaddee & Hystrix cristata & Spine, Burrow \& footprint & 0 & 0 & 1 \\
\hline 6 & Anubis baboon & Jaldeessa & Papio anubis & Observation & 0 & 0 & 1 \\
\hline 8 & Leopard & Qeeransa & Panthera pardus & Observation & 0 & 0 & 1 \\
\hline 9 & Menelik's bush & Borofa gurracha & Tragelaphus scriptus meneliki & Observation & 0 & 0 & 1 \\
\hline 10 & Black-back jackal & Sardiida dugda gurrraacha & Canis mesomelas & Observation & 1 & 1 & 0 \\
\hline 11 & Monkey & Qamalee & Cercopithecus aethiops & Observation & 0 & 0 & 1 \\
\hline 12 & Abyssinian Hare & Illeetti & Lepus capensis & Observation & 0 & 1 & 0 \\
\hline 13 & Aardvark & Awwaaldigessa & Orycteropus afer & Burrow \& R & 0 & 0 & 1 \\
\hline 14 & Bushbuck & Bosoonu & Tragelaphus scriptus & Observation & 0 & 0 & 1 \\
\hline
\end{tabular}




\begin{tabular}{|c|c|c|c|c|c|c|c|}
\hline \multirow{2}{*}{ S.No } & \multirow{2}{*}{ Common name } & \multirow{2}{*}{ Local name } & \multirow{2}{*}{ Scientific name } & \multirow{2}{*}{ Methods of identification } & \multicolumn{3}{|c|}{ Habitat types } \\
\hline & & & & & Erc & Af-al & Af-mo \\
\hline 15 & Guerza & Weennii & Colobus guereza percivali & Observation & 0 & 0 & 1 \\
\hline 16 & Spotted hyena & Waraabessa & Crocuta crocuta & Observation & 1 & 1 & 1 \\
\hline 17 & Klipspringer & Gicii & Oreotragus oreotragus & Observation & 1 & 1 & 0 \\
\hline 18 & $\begin{array}{l}\text { Ethiopian Rock } \\
\text { hyrax }\end{array}$ & Osolee & Procavia habessinicia & Secondary data source & 0 & 1 & 0 \\
\hline 19 & Mongoose & Amaa & Herpestes sanguine & Observation & 1 & 0 & 1 \\
\hline 20 & Serval cat & Deeroo & Felis serval & Secondary data source & 0 & 0 & 1 \\
\hline 21 & Zorilla & & Ictonyx striatus & Secondary data source & 1 & 0 & 0 \\
\hline 22 & Civet cat & Moor'ee & Civettictis civetta & Scent mark & 1 & 0 & 1 \\
\hline 23 & Caracal & Warbaa & Caracal caracal & Secondary data source & 1 & 0 & 0 \\
\hline 24 & Common jackal & Jeedala bakka maraa & Canis aureus & Secondary data source & 1 & 0 & 1 \\
\hline 25 & Honey badger & Amaa gaaguraa & Mellivoracapensis & Secondary data source & 0 & 0 & 1 \\
\hline 26 & Mongoose/Egyptian & Amaa & Herpestes inchneumon & Secondary data source & 0 & 0 & 1 \\
\hline 27 & Aard wolf & & Protelus cristatus & Secondary data source & 0 & 0 & 1 \\
\hline \multirow[t]{2}{*}{28} & Stark's hare & Illeettii & Lepus starki & Secondary data source & 0 & 1 & 0 \\
\hline & Total & & & & 13 & 9 & 20 \\
\hline
\end{tabular}

*Habitat types code: Erc=Ericaceous habitat, Af-al=Afro-alpine habitat,

Af-MO=Afro-Montane habitat, present $=1$, absent $=0$

Bohor reedbuck was the most dominant species in the area and it is about $27.8 \%$ and followed by warthog about $19.1 \%$. Totally 252 large mammals belongings to 28 species were recorded from three vegetation habitat types. About $28.97 \%$ mammals were recorded from ericaceous forest, $10.32 \%$ from afro-alpine forest and $60.32 \%$ from afro-montane (Woodland) forest were recorded (Table 3).

Table 3. Observation site and large mammals recorded via direct and indirect methods in Chilalo-Galema National Park.

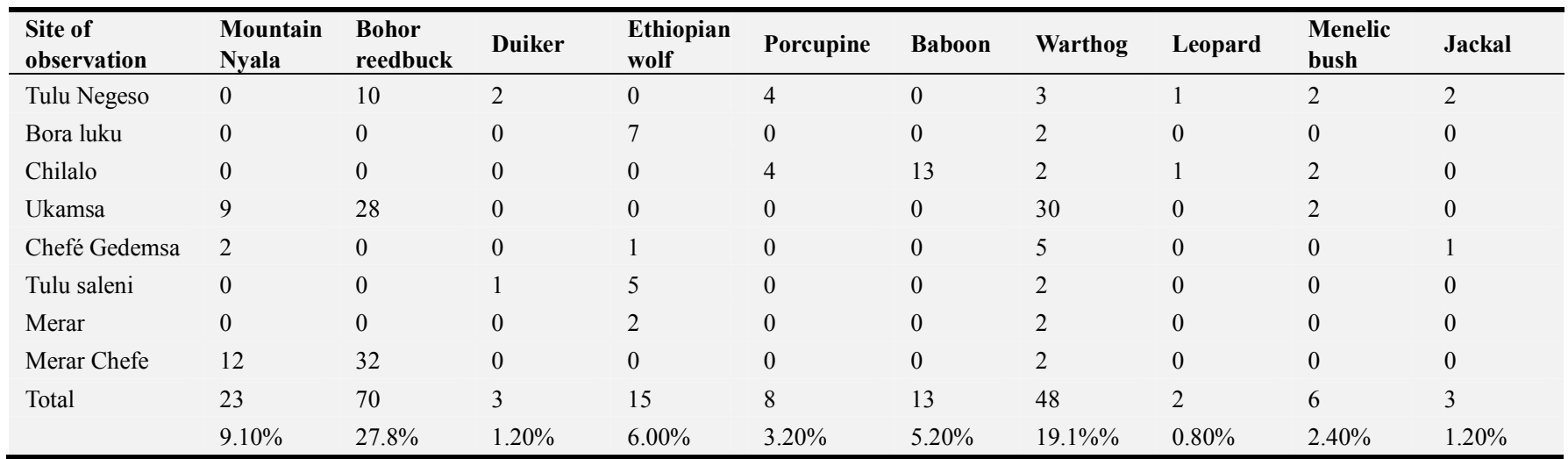

Table 3. Continued.

\begin{tabular}{|c|c|c|c|c|c|c|c|c|c|c|}
\hline $\begin{array}{l}\text { Site of } \\
\text { observation }\end{array}$ & Klipspringer & Monkey & Hare & Aardvark & $\begin{array}{l}\text { Bushbuck } \\
\text { common }\end{array}$ & $\begin{array}{l}\text { Colobus black } \\
\text { and white }\end{array}$ & $\begin{array}{l}\text { Civet } \\
\text { cat }\end{array}$ & Mongoose & Hyena & $\begin{array}{l}\text { Total } \\
\text { (N) }\end{array}$ \\
\hline Tulu Negeso & 0 & 0 & 0 & 2 & 0 & 0 & 1 & 0 & 2 & 29 \\
\hline Bora luku & 2 & 0 & 1 & 0 & 0 & 0 & 0 & 0 & 1 & 13 \\
\hline Chilalo & 0 & 7 & 0 & 2 & 1 & 6 & 0 & 0 & 4 & 42 \\
\hline Ukamsa & 0 & 0 & 0 & 2 & 1 & 0 & 1 & 4 & 5 & 82 \\
\hline Chefé Gedemsa & 0 & 0 & 1 & 0 & 0 & 0 & 0 & 0 & 3 & 13 \\
\hline Tulu saleni & 7 & 0 & 0 & 0 & 0 & 0 & 0 & 0 & 1 & 16 \\
\hline Merar & 3 & 0 & 0 & 0 & 0 & 0 & 0 & 2 & 1 & 10 \\
\hline Merar Chefe & 0 & 0 & 0 & 0 & 0 & 0 & 0 & 0 & 1 & 47 \\
\hline \multirow[t]{2}{*}{ Total } & 12 & 7 & 2 & 6 & 2 & 6 & 2 & 6 & 18 & 252 \\
\hline & $4.80 \%$ & $2.80 \%$ & $0.80 \%$ & $2.40 \%$ & $0.80 \%$ & $2.40 \%$ & $0.80 \%$ & $2.40 \%$ & $7.10 \%$ & $100 \%$ \\
\hline
\end{tabular}

\subsection{Species Distribution and Abundance of Large Mammalian Species Recorded Through Direct and Indirect Observation Indifferent Study Sites in Chilao-Galema National Park}

The three habitat types (eight study sites) with their species type, number of individual species (n), Total number of all species observed (N), Pi, Pi, ${ }^{2}$ LnPi \& PiLnPi are shown in (Table 4). 
Table 4. Species type, number of each species ( $n$ ), and total number of all species $(N)$ recorded through direct and indirect observation method in three habitat types.

\begin{tabular}{|c|c|c|c|c|c|c|c|c|c|c|}
\hline Habitat type & Study site & Species type & $\mathbf{n}$ & $\mathbf{N}$ & $\mathbf{n} / \mathbf{N}$ & $\mathbf{P i}$ & $\mathbf{P i}^{2}$ & $\ln P i$ & PilnPi & $\begin{array}{l}\text { H'(Index of } \\
\text { diversity) }\end{array}$ \\
\hline \multirow{16}{*}{ Afro-montane } & \multirow{16}{*}{$\begin{array}{l}\text { Chilao Ukamsa } \\
\text { \&Tulu Negeso }\end{array}$} & Bohor reedbuck & 38 & 152 & $38 / 152$ & 0.25 & 0.063 & -1.386 & -0.347 & \multirow{16}{*}{1.904} \\
\hline & & Red duiker & 2 & 152 & $2 / 152$ & 0.013 & 0 & -4.343 & -0.056 & \\
\hline & & Porcupine & 8 & 152 & $8 / 152$ & 0.053 & 0.003 & -2.937 & -0.156 & \\
\hline & & Warthog & 35 & 152 & $35 / 152$ & 0.23 & 0.053 & -1.469 & -0.338 & \\
\hline & & Leopard & 2 & 152 & $2 / 152$ & 0.013 & 0 & -4.343 & -0.056 & \\
\hline & & Menelik's bush buck & 6 & 152 & $6 / 152$ & 0.039 & 0.001 & -3.244 & -0.127 & \\
\hline & & Jackal & 2 & 152 & $2 / 152$ & 0.013 & 0 & -4.343 & -0.056 & \\
\hline & & Aardvark & 6 & 152 & $6 / 152$ & 0.039 & 0.001 & -3.244 & -0.127 & \\
\hline & & Civet cat & 2 & 152 & $2 / 152$ & 0.013 & 0 & -4.343 & -0.056 & \\
\hline & & Mountain nyala & 9 & 152 & $9 / 152$ & 0.059 & 0.004 & -2.83 & -0.167 & \\
\hline & & Mongoose & 4 & 152 & $4 / 152$ & 0.026 & 0.001 & -1.347 & -0.035 & \\
\hline & & Bush buck & 2 & 152 & $2 / 152$ & 0.013 & 0 & -4.343 & -0.056 & \\
\hline & & Hyaena & 10 & 152 & $10 / 152$ & 0.066 & 0.004 & -2.718 & -0.179 & \\
\hline & & Baboon & 13 & 152 & $13 / 152$ & 0.086 & 0.007 & -2.453 & -0.211 & \\
\hline & & Monkey & 7 & 152 & $7 / 152$ & 0.046 & 0.002 & -3.219 & -0.148 & \\
\hline & & Guereza & 6 & 152 & $6 / 152$ & 0.039 & 0.001 & -3.244 & -0.127 & \\
\hline \multirow{8}{*}{ Ericaceous } & \multirow{8}{*}{$\begin{array}{l}\text { Tulu saleni, } \\
\text { Meraro and } \\
\text { Merare cheffe }\end{array}$} & Red duiker & 1 & 73 & $1 / 73$ & 0.014 & 0 & -4.269 & -0.059 & \multirow{8}{*}{1.57} \\
\hline & & Ethiopian wolf & 7 & 73 & Jul-73 & 0.068 & 0.005 & -2.688 & -0.182 & \\
\hline & & Hyaena & 3 & 73 & $3 / 73$ & 0.027 & 0.001 & -3.612 & -0.097 & \\
\hline & & Klipspringer & 10 & 73 & $10 / 73$ & 0.137 & 0.019 & -1.988 & -0.272 & \\
\hline & & Warthog & 6 & 73 & $6 / 73$ & 0.082 & 0.007 & -2.501 & -0.205 & \\
\hline & & Mountain nyala & 12 & 73 & $12 / 73$ & 0.164 & 0.027 & -1.808 & -0.296 & \\
\hline & & Bohor reed buck & 32 & 73 & $32 / 73$ & 0.438 & 0.192 & -0.826 & -0.361 & \\
\hline & & Mongoose & 2 & 73 & $2 / 73$ & 0.027 & 0.001 & -3.612 & -0.098 & \\
\hline \multirow{7}{*}{ Afro-alpine } & \multirow{7}{*}{$\begin{array}{l}\text { Bora luku and } \\
\text { Cheffe } \\
\text { gedemsa }\end{array}$} & Mountain nyala & 2 & 27 & $2 / 73$ & 0.074 & 0.005 & -2.603 & -0.192 & \multirow{7}{*}{1.809} \\
\hline & & Warthog & 7 & 27 & 27-Jul & 0.259 & 0.067 & -1.35 & -0.349 & \\
\hline & & Jackal & 1 & 27 & $1 / 73$ & 0.037 & 0.001 & -3.296 & -0.121 & \\
\hline & & Abyssinia hare & 2 & 27 & $2 / 73$ & 0.074 & 0.005 & -2.603 & -0.192 & \\
\hline & & Ethiopian wolf & 8 & 27 & $8 / 73$ & 0.296 & 0.087 & -1.217 & -0.36 & \\
\hline & & Hyaena & 5 & 27 & $4 / 73$ & 0.185 & 0.034 & -1.687 & -0.312 & \\
\hline & & Klipspringer & 2 & 27 & $2 / 73$ & 0.074 & 0.005 & -2.603 & -0.192 & \\
\hline
\end{tabular}

\subsection{Diversity Indices of Large Mammalian Species in Chilalo-Galema National Park Block}

A total of 28 large mammalian species were identified and recorded. Three habitat types Ericaceous forest, Afromontane (Woodland) forest and Afro-alpine forest were represented by 8,16 , and 8 species respectively. Diversity indices $\left(\mathrm{H}^{\prime}\right)$ and evenness $(\mathrm{J})$ of large mammal species along the three habitat types were shown in (Table 6). Afromontane (Woodland) forest had the highest diversity index (1.904) and evenness (0.686), thus afro-montane forest had more diverse community and species are evenly distributed, whereas the lowest diversity index (1.57) and evenness $(0.755)$ was recorded in ericaceous habitat this showed that ericaceous habitat had less diverse community and evenly distributed species (Table 5).

Table 5. Diversity indices ( $\left.H^{\prime}\right)$ and evenness $(J)$ of mammals in Chilalo-Galema National Park.

\begin{tabular}{|c|c|c|c|c|c|c|c|c|}
\hline Habitat type & & Study site & $\begin{array}{l}\text { Number } \\
\text { of } \\
\text { species } \\
\text { (s) }\end{array}$ & $\begin{array}{l}\text { Total number } \\
\text { of species in } \\
\text { habitat }(\mathrm{N})\end{array}$ & $\begin{array}{l}- \\
\mathbf{i}=1\end{array}$ & $H_{\text {max }}^{\prime}=\ln (s)$ & $\begin{array}{l}\text { H'(Index } \\
\text { of } \\
\text { diversity) }\end{array}$ & $\begin{array}{l}\text { J } \\
\text { (evenness) }\end{array}$ \\
\hline Afro-montane & & Tullu Negeso, Ukamsa and chilalo & 16 & 152 & -1.904 & 2.772 & 1.904 & 0.686 \\
\hline Ericaceous forest & & $\begin{array}{l}\text { Tulu saleni Merarie chef and } \\
\text { Meraro }\end{array}$ & 8 & 73 & -1.57 & 2.079 & 1.57 & 0.755 \\
\hline $\begin{array}{l}\text { Afro-alpine and } \\
\text { forest }\end{array}$ & Ericaceous & Boraluku and Cheffe gedemsa & 8 & 27 & -1.809 & 2.079 & 1.809 & 0.87 \\
\hline
\end{tabular}

There were 16 species in Afro-montane habitat, eight species in ericaceous and 8 species in afro-alpine habitat. Between three habitats they have 3 species in common.

Simpson's similarity index $=3 * 3 / 16+8+8=9 / 32=0.281$
Simpson similarity index (SI) of large mammalian species among three habitats (eight study site area) was 0.281 . This indicated that $28.1 \%$ of the species were common for all three habitats. The highest similarity occurrence between 
ericaceous against afro-alpine (0.625) and followed by ericaceous against afro-montane (woodland) $(0.5)$. Whereas the lowest similarity occurrence $(0.333)$ was between afro- alpine against afro-montane (woodland). The Simpson's similarity index of large mammals for three habitat types is given in (Table 6).

Table 6. Simpson similarity index (SI) of large mammalian species among three habitat types.

\begin{tabular}{llll}
\hline Habitat (vegetation type) & Species number & Common species & Simpson's similarity \\
\hline Ericaceous against afro-montane & $8 \& 16$ & 6 & 0.5 \\
Ericaceous against afro-alpine & $8 \& 8$ & 5 & 0.625 \\
Afro-montane against afro-alpine & $16 \& 8$ & 4 & 0.333 \\
\hline
\end{tabular}

\section{Discussion}

In the current study about 16 species of large mammals were identified and recorded. The habitat types contributed a lot for the distribution of large mammals in the area. Predominantly there are three habitat types in the area namely, afro-alpine, afro-montane and ericaceous. Accordingly, 16 species were distributed in afro-alpine habitat, 8 species in afro-montane and 8 species in ericaceous.

The possible reason for this distribution of large mammalian species in the area may be due to the availability of sufficient resources such as food and water. The result of this study agrees with studies conducted in different areas both in Ethiopia and in other African countries where large mammals were abundantly distributed in different habitat types. For instance the current study agrees with the findings of [27] where he recorded 23 species in Arawale National Reserve, Kenya; [28] recorded 23 species in Sime Darby, Liberia; [29] recorded 19 species in Wendo Genet, Ethiopia, [30], recorded 25 species in Harena forest, Ethiopia, and [24] recorded 23 species in the moist semi-deciduous forest of Ghana.

The species of mammals detected through direct observation were mountain nyala (Tragelaphus buxtoni), bohor reedbuck (Redunca redunca), red duiker (Cephalophus natelensis), Ethiopian wolf (Canis simensis), anubis baboon (Papio anubis), warthog (Phacochoerus africanus), Menelik's bushback (Tragelaphus scriptus meneliki), black-back jackal (Canis mesomelas), Monkey (Cercopithecus aethiops), Abyssinian hare (Lepus capensis), bushbuck common

(Tragelaphus scriptus), guereza (Colobus guereza percivali), spotted hyena (Crocuta crocuta), Leopard (Panthera pardus), mongoose (Herpestes sanguine) and klipspringer (Oreotragus oreotragus).

Nocturnal species such as civet cat (Civettictis civetta), aardvark (Orycteropus afer) and porcupine (Hystric crista) were detected through indirect evidences such as foot print, burrow, spine and scent marks. Secondary data source from Arsi Mountains National Park indicated the that there are also different species such as serval cat (Felis serval), zorilla (Ictonyx striatus), caracal (Caracal caracal), scrub hare (Lepus fagani), common jackal (Canis aureus), honey badger (Mellivora capensis), mongoose/Egyptian (Herpestes inchneumon), aard wolf (Protelus cristatus) and Ethiopian rock hyrax (Procavia habessinicia) in the Chilalo-Galema Mountain National Park.

Primates species belong to the Family Cercopithecidae and Colobidae were identified in the woodland mixed with ericaceous forest through direct observation. Vervet monkey (Cercopithecus aethiops) and anubis baboon (Papio anubis) were observed at the edge of the forest closer to human dwelling areas and at the center of the natural forest. The location of the species at the edge of the forest might be associated with cultivated crops surrounding farmers. The result of this study agrees with [31]. Colobus monkey (Colobus guereza) was observed in the afro-mountain forest specifically on long trees. This might be associated with tree fruits used as food and height of the trees might secure them from danger. This study agrees with [32].

Among 252 mammals observed and recorded in ChilaloGalema Mountains about $26(10.10 \%)$ belong to order primates. From local community elders it was confirmed that primate population has been declining because the woodland was completely changed to agricultural activities and the natural forest which was home of these wildlife species was deforested, they have no place to live, so that either they have to migrate to other places or starved and died. Order Carnivora had the highest number of family and species with 6 and 13 respectively. Among total of 252 observed and recorded mammals about $50(19.38 \%)$ belongs to order Carnivora. Among the carnivores, about 18 (36\%) was spotted hyena. Hyena (Crocuta crocuta) was the most common mammals of order carnivora which was recorded in all habitat types such as afro-montane forest, ericaceous forest and afro-alpine forest. The present study agrees with [33] that he recorded spotted hyenas in the Bale Mountains, which is more $4,000 \mathrm{~m}$ high with altitude.

Besides, serval cat (Felis serval), zorilla (Ictonyx striatus), caracal (Caracal caracal), common jackal (Canis aureus), honey badger (Mellivor acapensis), mongoose/Egyptian (Herpestes inchneumon) and aard wolf (Protelus cristatus) were also observed and recorded in Chilalo-Galema National Park. Regarding the habitat type they are found in Ericaceous and woodland forest. Ethiopian wolf which is endemic and usually depend on prey rodents was found in Afro-alpine forest of the Chilalo Galema Mountains. This study agreed with [16] Chilalo-Galama Mountain they found the second largest population of the Ethiopian wolf next to the Bale Mountains with $572 \mathrm{~km}^{2}$ of suitable habitat.

Order artiodactyla have two families (Suidae and Bovidae) and seven species warthog (Phacochoerus africanus), red duiker (Sylvicapra grimmia), klipspringer (Oreotragus oreotragus), bohor reedbuck (Redunca redunca), bush buck (Tragelaphus scriptus), Menelik's bush (Tragelaphus scriptus meneliki) and mountain nyala (Tragelaphus buxtoni). Out of 
the 252 recorded mammals in this study $26(10.08 \%)$ were order primates, $50(19.38 \%)$ belongs to order carnivora, 1 $(0.39 \%)$ belongs to order hyracoidea, $6(2.33 \%)$ belongs to order tubulidentata, $164(63.57 \%)$ belongs to artiodactyla, 8 (3.102 and $(0.78 \%) \%)$ were rodentia and lagomorpha respectively. The current study indicated that the mammalian fauna of the Chilalo Galema Mountain (ChGM) was dominated by two groups of mammalian orders (the most abundant orders); namely, order artiodactyla and order carnivora, composed of $164(63.57 \%)$ and 50 (19.38\%) respectively. The least abundant was order hyracoidean 1 $(0.39 \%)$ mammals. Endemic species like Mountain nyala (Tragelaphus buxtoni) and Menelik's bushbuck (Tragelaphus scriptus meneliki)) belongs to order artiodactyla and Ethipian wolves (Canis simensis) were also found in Chilalo Galema Mountains which is endemic to Ethiopia.

From recorded 164 mammals of Order artiodactyla family Bovidae has 6 species that is $70(42.68 \%)$ were bohor reedbucks (Redunca redunca), 23 (14.02\%) was Mountain nyala (Tragelaphus buxtoni), $12(7.32 \%)$ was Klipspringer (Oreotragus oreotragus), 6 (3.66\%) was Menelik's bush (Tragelaphus scriptus meneliki), 3 (1.83\%) was Red duiker (Sylvicapra grimmia) and $2(1.22 \%)$ was common bush buck (Tragelaphus scriptus meneliki), family Suidae has one species which is 48 (29.27\%) was warthogs (Phacochoerus africanus). This showed that the most dominant and abundant species in Chilalo Galema Mountains range was bohor reedbucks (Redunca redunca) and followed by warthogs (Phacochoerus africanus). This species appeared to be more concentrated in ericaceous forest and afro-montane (plantation) forest habitat. The species were more frequently observed during the mid-day and late afternoon. This might prevent the species from nocturnal predators.

Order tubulidentata has one family (Orycteropodidae) and one species (Orycteropus afer). Among mammalian species recorded in Chilalo Galema Mountains, aardvark (Orycteropus afer) was the only representatives of the Order Tubulidentata detected in the study area. Aardvark was a nocturnal animal and shelters during the day in deep burrows. The current study agrees with [34] that aardvark has been overlooked in Ethiopia due to its nocturnal habits and its shelters during the day in deep burrows. Order rodentia has one family (Hystricida) and one species (Hystrix cristata). Porcupine (Hystrix cristata) is a nocturnal mammal that belongs to order rodentia. Order hyracoidea has one family (Procaviidae) and one species, Ethiopian rock hyrax (Procavia habessinica). Order lagomorpha has one family (leporidae) and two species of abyssinian hare (Lepus capensis) and scrub hare (Lepus fagani).

During this study it was observed that the forest which serves wildlife as home, sources of food and other resources has been deforested, as a result the wildlife in the area would be at risk of local extinction, and wildlife conflict was also observed because animals raid crops. Thus, conservation measures will be taken by the local community as well as by the government to safeguard the mammalian species in the study area.

\section{Conclusion and Recommendations}

\subsection{Conclusion}

This study revealed that Chilalo Galema Mountains National Park is home for endangered and endemic mammals like critically endangered endemic Ethiopian wolf, endangered and endemic Mountain nyala and Menelik's bushbuck. The present study also identified and documented mammalian species of Chilalo Galema Mountains National Park and gave base line information about their presence. The distribution and abundance of mammalian species in Park varied based on vegetation types and altitudinal variation. For example, guereza, anubis baboon, monkey, bushbuck, Menelik's bush buck and leopard were frequently observed in woodland habitat. The numbers of large mammalian species recorded during the entire study period from Chilalo Galema Mountains forest were compared with several other studies conducted in Ethiopia and other African countries. The area harbors many large mammalian species, birds and other wildlife species. Therefore, it can be considered as important center for conservation of the wildlife species and used as tourist destination area.

\subsection{Recommendations}

Based on the findings of the current study the following recommendations were suggested:

1. The present study confirms that the Chilalo Galema Mountain forest contains rich diversity of mammals and hence its conservation and biodiversity documentation efforts should be considered critically

2. To conserve the wildlife in the park, threats such as encroachment, livestock grazing, grass cutting, environmental degradation, poaching and deforestation should be minimized.

3. To reduce human-wildlife conflict, using physical barriers such as fences and also buffer zones should be demarcated.

4. Promote community-based solutions to prevent and manage human-wildlife conflicts.

\section{Conflict of Interest}

Authors have no conflict of interest

\section{Acknowledgements}

We would like to thank Adama Science and Technology University for financial support.

We would also like to thank Biology Department for facilitating the entire research work.

\section{References}

[1] Kingdom, J., (1971). East Africa Mountains: An Atlas of Evolution in Africa. Vol. I. Academic Press, London, 242 PP. 
[2] Yalden, D. W., Largen, M. J. and Kock, D. (1992). Catalogue of the mammals of Ethiopia. Primate Monit. Zool. (NS) Suppl. 9: $1-52$.

[3] Mesele Admassu, Yosef Mamo and Afework Bekele (2011). Damage caused by large mammals in Wonji shoa Sugar cane plantation, Ethiopia, J. Agri. Biol. Sci., 2 (6): 151-157.

[4] Hillman, J. C. (1993). Ethiopia: Compendium of Wildlife Conservation Information. New York Zoological Society and Ethiopian Wildlife Conservation Organization, Addis Ababa PP. 1-18.

[5] Tekalign, W. (2006). Distribution, population status and feeding behavior of oribi (Ourebia ourebi) in Senkelle Swayne's Hartebeest Sanctuary (SSHS).

[6] Yosef Mamo, Afework Bekele \& Girma Mengesha (2012 a). Habitat use of mountain Nyla (Tragelaphus buxtoni, Lyddeker, 1911) in the Bale Mountains National Park. Ethiopia. Int. J. Biodivers Conserv. 4: 642-651.

[7] Idris, A. B., Gonzaga, A. D., Zaneedarwaty, N. N., Hasnah, B. T. and Natasha, B. Y. (2001). Does habitat disturbance has adverse effect on the diversity of parasitoid community? $J$. Biol. Sci., 1: 1040-104.

[8] Maan, M. A. and Chaudhry, A. A. (2001). Wildlife Diversity in the Punjab (Pakistan). J. Bioloical Sci., 1: 417-420.

[9] Gabol, K., Mehmood, K, Yasmin, N, Tariq, R. M, and Tabassum, R. (2005). Distribution, status of migratory and resident waterfowls of Drigh lake (Sindh) wildlife sanctuary. Int. J. Zool. Res., 1: 37-40.

[10] Gundogdu, E. (2011). Population size, structure and behaviors of wild goat in cehennemdere wildlife improvement area. Asian J. Anim. Vet. Adv, 6: 555-563.

[11] Evangelista, P., Swartzinski, P. and Waltermire, R, (2007). A profile of the mountain Nyala (Traglaphus buxtoni). Retrieved from www.Africanindaba Accessed on December, 20, 2018, pp: 46.

[12] IUCN (1996). IUCN Red List of threatened Animals, IUCN. Gland, Switzerland.

[13] Purvis, A., Gittleman, J. L., Cowlishaw and G., Mace, G. M. (2000). Predicting extinction risk in declining species. Proceedings of the Royal Society of London B 267, 19471952. Promislow, D. E. L. 1993 On size and survival: progress and pitfalls in the allometry of life span. J. Gerontol. 48, B115-B123. (doi: 10.1093/geronj/48.4.B115).

[14] Cardillo, M., Mace, G. M., Jones, K. E., Bielby, J., BinindaEmonds, O. R. P., Sechrest, W., Orme, C. D. L. and Purvis, A. (2005). Multiple Causes of High Extinction Risk in Large Mammal Species. Scien press. www.sciencexpress.org/pp.

[15] Gottelli, D. and Sillero-Zubiri, C. (1992). The Ethiopian wolf, an endangered endemic canid. Oryx. 26: 2005-214.

[16] Malcolm, J. R. and Sillero-Zubiri, C. (1997). The Ethiopian wolf; Distribution and population status. In: The Ethiopian wolf Status Survey and Conservation Action Plan, 12- 25 pp, (Sillero-Zubiri, C. Macdonald D. W. eds). IUCN, Gland and Cambridge.

[17] Alemayehu Mengistu (1975). Grassland Condition in the Chilalo Awraja, Ethiopia. CADU Publications, No. 112, Asella, $25 \mathrm{pp}$.
[18] APEDO and ABRDP (2004). Atlas of Arsi Zone. APEDO (Arsi Zone Planning and Economic Development Office) and ABRDP (Arsi-Bale Rural Development Project), Asella, 35 pp.

[19] Yimer, D. (2008). Mammalian diversity in Mazie National Park, Ethiopia. M.Sc. Thesis, Addis Ababa University.

[20] Norton-Griffiths, M. (1978). Counting Animals. 2nd ed. Serengeti Monitoring Programme. African Wildlife Foundation, Nairobi, Kenya.

[21] Seber, G. A. F. (1982). The Estimation of Animal Abundance and Related Parameters. 2nd ed. MacMillan, New York.

[22] Alden, P. E., Estes, R. D., Schlitter, D. and McBride, B. (1995). Field Guide to African Wildlife. Alfred, A. Knopf, Inc. New York, 443-633.

[23] Lee, M. E., Alonso, A., Dallmeier, F. P., Cambell and Pauwels, O. S. G. (2006). The Gambian complex of protected areas: An illustration of Gabon's biodiversity. In: Gamba, Gabon: Biodiversity of an Equatorial African Rainforest, 417_423 pp, (Alonso, A., Lee, M. E. Cambell, P., Pauwels, O. S. G. and Dallmeier, F., eds.). Washington: Bulletin of the biological society No. 12 .

[24] Ofori, B. Y., Attuquayefio, D. K. and Owusu, E. H. (2012). Ecological status of large mammals of a moist semi-deciduous forest of Ghana: implications for wildlife conservation. $J$. Biodiver. Enviro. Sci. 2: 28-37.

[25] Shannon. G. E. and Weaver. W. (1949). The Mathematical Theory of Communication. Chicago: University of Illinois Press.

[26] Flower. J. and Coher. L. O. (1990) Practical Statistics for Field Biology. Chi Chester: John Wiley and Sons.

[27] Njoroge, P., Yego, R., Muchane, M., Githiru, M., Njeri, T. and Giani, A. (2009). A survey of the large and medium sized mammals of Arawale national reserve, Kenya. J. East Afr. Nat. History 98: 119-128.

[28] Bene, J. K., Bitty, E. A., Bohoussou, K. H., Abedilartey, M., Gamys, J. and Soribah, P.A.J. (2013). Current conservation status of large mammals in Sime Darby oil palm concession in Liberia. Global Institute for Research \& Education. 2: 93-102.

[29] Zerhun Girma., Yosef Mamo and Mateos Ersado (2012). Species composition, distribution and Relative abundance of large mammals in and around Wondo Genet Forest Patch, Southern Ethiopia. Asian J. Appl. Sci. 5: 538-551.

[30] Afework Bekele and Leirs, H. (1997). Population ecology of rodents of maize field and grassland in central Ethiopia. $J$. Belg. Zool. 127: 39-48.

[31] Aramde Fetene., Girma Mengesha and Tsegaye Bbekele (2011). Spatial distribution and habitat preferences of selected large mammalian species in the NechSar National Park (NSNP), Ethiopia. Nature Sci. 9: 80-90.

[32] Yalden, D. W. and Largen, M. J. (1976). Endemic mammals of Ethiopia. Mamm. Rev. 22: 115-150.

[33] Sillero-Zubiri, C. (1996). Records of Honey Badger Mellivora capensis in Afroalpine habitats, above 4,000 m. Mammalia, 60: 323-325.

[34] Yalden, D. W.; Largen, M. J. \& Kock, D. (1986). Catalogue of the mammals of Ethiopia 6. Perissodactyla, proboscidea, hyracoidea, lagomorpha, tubulidentata, sirenia and cetacea. Monitore Zoologico Italiano, n.s. supplemento XXI, 4: 31-103. 